\title{
A Visual Enhancement Quality of Digital Medical Image Based on Bat Optimization
}

\author{
Kholood N. Hussin", Ali K. Nahar $\mathbb{D}^{\text {, Hussain K. Khleaf }}$ \\ Electrical Engineering Dept., University of Technology-Iraq, Alsina'a street, 10066 Baghdad, Iraq. \\ *Corresponding author Email: eee.19.31@grad.uotecgnology.edu.iq
}

\section{H I G H L I G H T S}

- This work proposes the Bat algorithm to denoise the medical image with different types of noise.

- Use several types of noise such as Gaussian noise, salt \& pepper noise, speckle noise.

- Bat algorithm achieved the best results in noise removal and enhance medical images.

- The proposed Bat optimization image denoising achieves better RMSE and PSNR values than other techniques (Gaussian filter, median filter, bilateral filter, and wiener filter).

- Observed the PSNR performance of the proposed method $(60.6,55.6,64.9,63.6)$ Gaussian noise, salt-and-pepper noise, speckle noise, and Poisson noise on order.

\section{A R T I C L E I N F O}

Handling editor: Ivan A. Hashim

Keywords:

Medical images

De-noising

BAT algorithm

PSNR

Wiener filter

\author{
A B S T R A C T
}

Improve medical image visualization is a critical preliminary step before further imagery processing like analyzing texture, extracting features, and segmentation. Imagery noises in medical images are frequently occurred as a consequence of different artificial processes such as acquisition, sending and receiving, and storing \& retrieving processes. As a result, the quality of image visualization is degraded. Therefore, a de-noise process is important in order to maintain good image quality for medical purposes. In this paper, medical image enhancement aims to de-noise as much as possible while maintaining detailed features and edges. This work employed an optimization algorithm called "Bat" to enhance the quality of the medical images and also compare it with other methods such as Gaussian filter, median filter, and bilateral and Wiener filter. Obtained image quality was evaluated using range of reference metrics, like, peak signal to noise ratio (PSNR), mean square error (MSE), structural similarity index measure (SSIM), and signal to noise ratio (SNR). Bat algorithm achieved the best PSNR, SNR, MSE, SSIM values compared to other filters. Findings presented in this research showed that the PSNR performance of the proposed method is $(60.6$, $55.6,64.9,63.6)$, MSE is $(1.125,1.43,2.95,1.15)$, Gaussian noise, salt-andpepper noise, speckle noise, Poisson noise on order.

\section{Introduction}

Image processing is a developing field, with novel applications that develop at a fast-growing pace. Digital imagery is a computerized-simulated field with expanded applications ranging from the entertainment industry to the space program [1]. The fascinating characteristics of such a revolution of information can be referred to as the capability of sending and receiving complex data that goes beyond usually written texts. The transmission of visual information in the shape of a digital image becomes one of the most important means of communicating in the twenty-first century [2]. Image processing is process signals in any form, for which the input represents the image, like video frames or photos, and image processing outputs an image or a group of image-related properties or parameters [3, 20]. The objective of this research is de-noising images based on the Bat algorithm to increase the visual quality and keep the edge of medical images and build the adaptive model on the different types of medical images. The performance of developed algorithms/methods is compared to the current methods regarding peak signal-to-noise ratio (PSNR), Mean Square Error (MSE), structural similarity index measure (SSIM), and SNR (Signal to Noise Ratio). Effective image de-noising is considered a significant matter. The paper is organized as follows: the 
second section included a review of the related work with a brief description of noise and Bat optimization algorithm. Then, the work methodology and discuss the results and conclusion were presented in the remaining sections.

\section{Related Work and Background}

\subsection{Related work}

De-noising process of medical images was recently investigated under various research strategies. For example [3] proposed an algorithm adopted for enhancing a specific original input image in the field of wavelets, and results were verified using the values of PSNR. The algorithm is also employed to image contrast equalization that improves the values of PSNR and enhances images. In [4], proposed Advanced Adaptive Algorithm to enhance dark medical images. This approach relies primarily upon adaptive gamma correction by using Singular Value Decomposition (SVD) and discrete wavelet transform (DWT). Dissimilar kinds of non-contrast computed tomography imaging are regarded for evaluating the proposed algorithm's performance related to contrast enhancement, which depends upon adaptive gamma correction by using Singular Value Decomposition and discrete wavelet transform. In [5] low-contrast effects often degrade satellite image quality. Thus, various researches are conducted to treat this type of effect. However, the gap of knowledge of this field of work is still not well addressed, no conclusion has been obtained yet, and a range of scientific questions needs to be studied thoroughly.

Moreover, the original BCETP uses three coefficients to determine a parabolic function. On the other hand, the proposed ABCETP functions through calculating an adjusted parabolic function using two real coefficients and an adjusted coefficient. In addition, it uses two different techniques for producing results with adequate quality. The proposed technique was checked with many actual contrast distortion images, and a comparison is made to different methods of contrast improvement, and the evaluation of the result is conducted using two dedicated quality assessment measures. Furthermore, Experimental results obtained by performing many comparisons and experiments illustrate the preference of this method, producing better quality images and outperforming other comparison methods in many properties. In [6] a proposed algorithm that can enhance the vaginoscopy images' visual quality of bitches via the use of Discrete Cosine Transform (DCT), (DWT), and SVD. The input image decomposes in four frequency bands by the use of DWT. The converted image's low-frequency band is converted back to high-frequency (HF) discrete cosine transforms coefficients and low-frequency (LF) discrete cosine transforms coefficients by using discrete cosine transform. Singular Value Decomposition forms a new LF band. In 2020, [7] proposed a new particle swarm optimized texture-based histogram equalization (PSOTHE) technique for enhancing the contrast of brain images of Magnetic resonance imaging.

Generally, the medical image is low - contrast and noise sources can affect it. This can strongly influence the accuracy of diagnostic processes. To tackle this problem, the influence of this type of artifact must be eliminated or reduced. Also, it is essential to optimize the contrast and reduce the loss of information. A new multi-objective fitness function is suggested for obtaining the image having the best balance and enhancement, it contains four dissimilar objective functions like edge, entropy, contrast, contents, peak signal to noise ratio, and every equivalent weightage. In [8], introduce a design and application of a technique known as Biogeography-based Optimization algorithm with Elite Learning BBO-EL algorithm for solving the troubles related to registering multimedia medical imaging. In [9], a novel krill herd-based optimized contrast and sharp edge enhancement framework were introduced to optimize the medical image. In this work, KH-RHE frameworks are designed to choose the variant and flexible plateau limit automatically according to X-ray images, magnetic resonance imaging, mammogram, and computerized tomography, needless to any intervention from a human. The processes of redistribution and clipping are combined with general histogram equalization (GHE) for controlling over-enhancement by limiting the rate of improvement and increasing dynamic image grayscale separately.

\subsection{Sources of Noise and Mathematical Representation of Noise}

In the processes of acquiring, transmitting, storing, and retrieving, noise image contaminates image signal. In various engineering applications, acquisition noise is commonly very low-contrast, and it is insignificant. The main reason is that the high quality of the sensors [10]. On the other hand, acquisition noise may play a significant role in specific applications, such as biomedical devices, remote sensing, and so on. However, the critical explanation in this method is that the image acquisition system is made up of a transmitter tube. AWGN, SPN, and mathematical formula were explained below Gaussian noise equally distribute over signal, that is meaning that every pixel in a noisy image represents real pixel values sum and random Gaussian distributed noise value can be expressed by equation (1) [11].

$$
F_{\text {awgn }}=f(x, y)+\eta_{G}(x, y)
$$

Where $\mathrm{n}_{G_{G}}$ a Random variable having Gaussian probability distribution with a bell is shaped probability distribution function expressed by in equation (2).

$$
F(g)=\frac{1}{\sqrt{2 \pi \sigma^{2}}} e^{-(g-m)^{2} / 2 \sigma^{2}}
$$

Where $\mathrm{g}$ is representing the grayscale, $\mathrm{m}$ represents the average or mean of the function, and $\sigma$ represents standard noise deviation. 


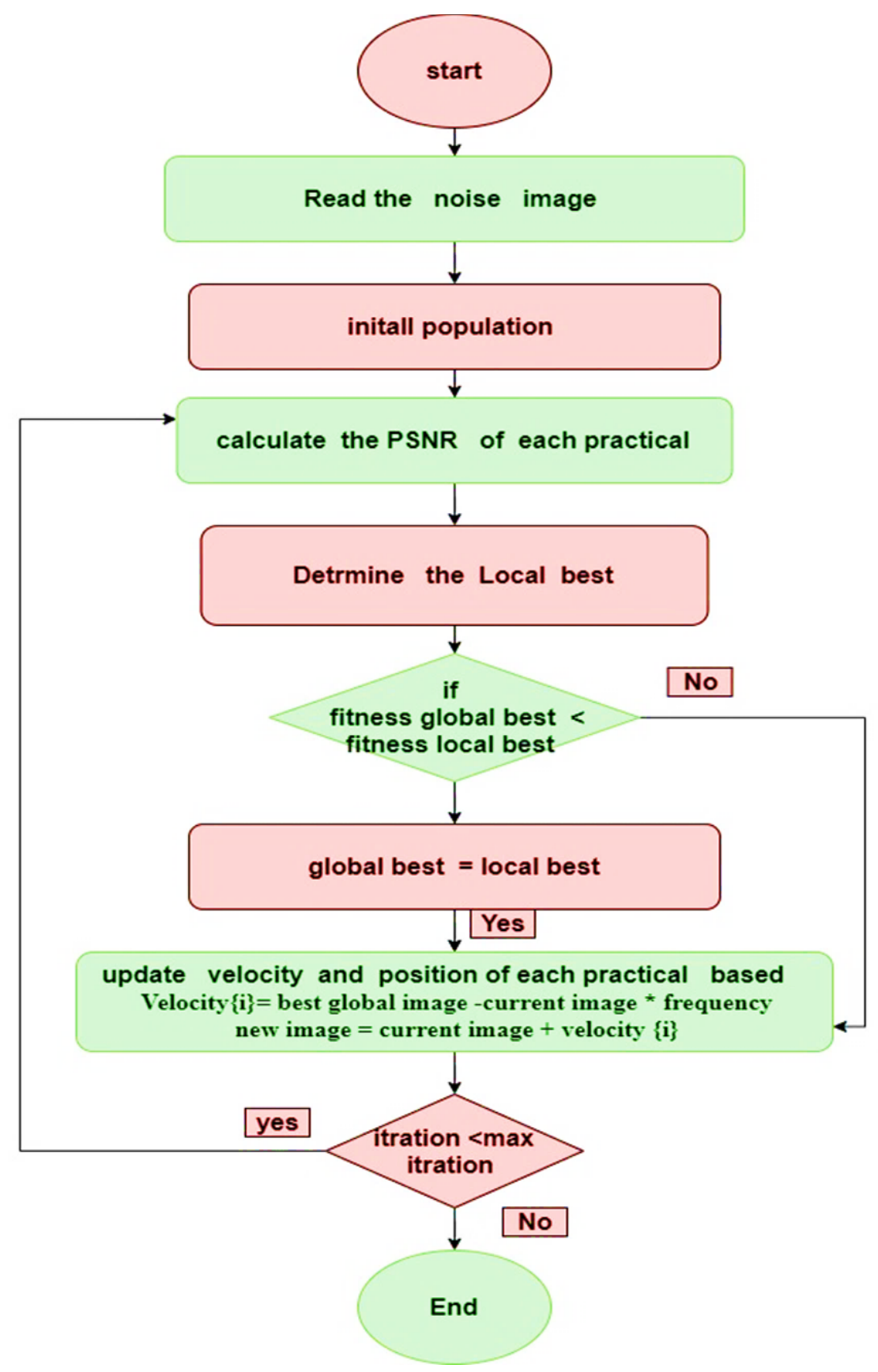

Figure 1: The flowchart of Denise of the proposed method (Bat Algorithm)

\section{Simulink Result and Discussion}

In this work, apply number filters and implemented us (MATLAB R2018b) and tested four types of noise: Salt and Pepper, Gaussian, Poisson noise, and Speckle noise. The de-noising image uses the Bat optimization, median filter, wiener filter, bilateral filter, and Gaussian filter. The work tests several medical digital images for different types such as MRI, CT, Xray, ultrasound images in a different format ('.jpg', '.png', '.bmp'). The results of our proposed methods that use the Bat optimization algorithm value for image de-noising have been compared with Gaussian filter, wiener filter, bilateral filter, and median filter with a different type of noise as shown in Figure 2, Figure 3, Figure 4, Figure 5.

In Analysis Results our experiment, we evolution the proposed de-noising the medical image based on bat optimization and compared with other filters test on the different type of medical image (x-ray, CT, MRI, ultrasound) by calculating the performance matrix such as PSNR, SNR, MSE, SSIM as shown in Table I, Table II, Table III, Table IV.

From Table, I show the power of the proposed technique to de-noising the Gaussian noise from a medical image when compared with other technique and the proposed method achieve 60.6 the value of PSNR but the second-best filter (Wiener filter) achieves the 26.2 PSNR 
Table 1: The performance measures of the proposed method and compare with other techniques for Gaussian noise removal

\begin{tabular}{lllll}
\hline Filter & PSNR & SNR & MES & SSIM \\
\hline Gaussfilt & 24.27133 & 14.54475 & 12.33344 & 0.416964 \\
Bilatfilt & 23.74722 & 14.08337 & 13.5678 & 0.405142 \\
Wiener & 26.24699 & 16.43792 & 11.58258 & 0.519622 \\
Bat algorithm (proposed) & 60.60919 & 50.70034 & 1.1258 & 0.999248 \\
Medfilt & 26.35195 & 16.47355 & 10.31749 & 0.578153 \\
Noise image & 14.5032 & 11.74502 & 27.5289 & 0.4660 \\
\hline
\end{tabular}
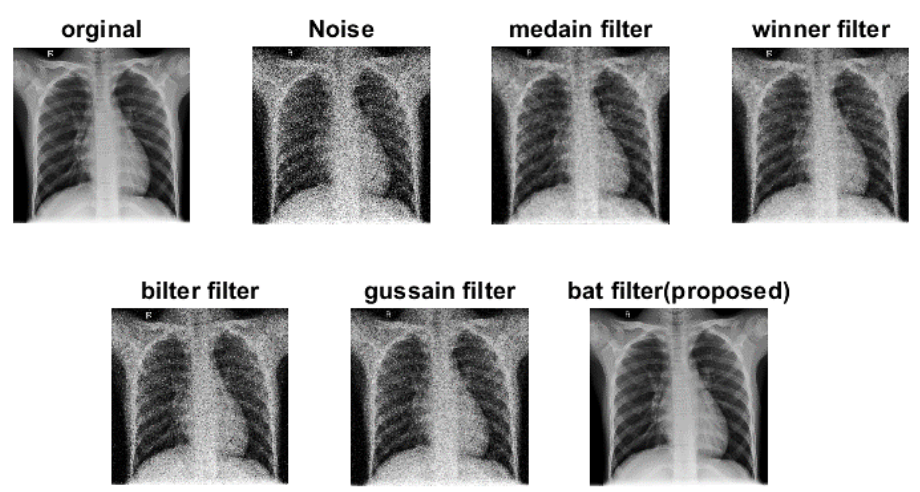

Figure 2: The X-Ray image enhancement by the proposed Bat algorithm compare with other techniques when adding gaussian noise
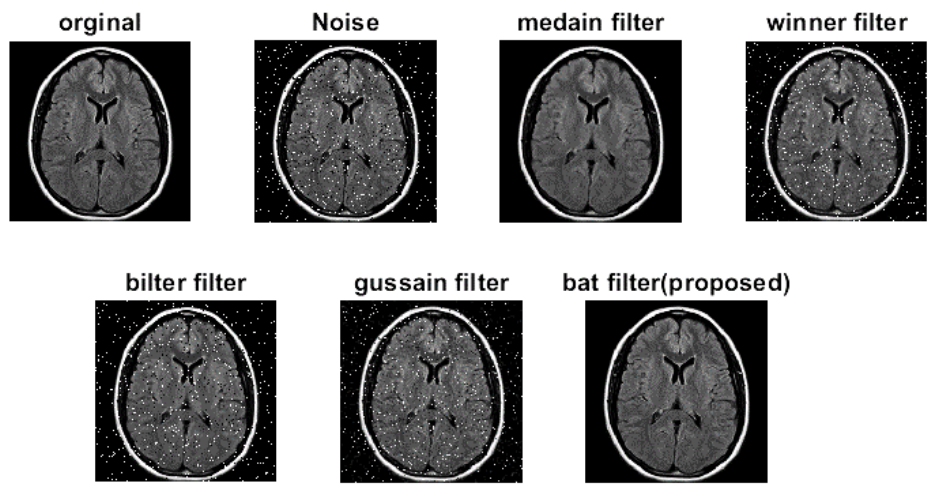

Figure 3: The MRI medical image enhancement by the proposed Bat algorithm and compare with other techniques when adding salt and pepper noise

Table 2: The performance measures of the proposed method and compare with other techniques for salt\& pepper noise removal

\begin{tabular}{lllll}
\hline Filter & PSNR & SNR & MES & SSIM \\
\hline Gaussfilt & 20.86639 & 11.40983 & 12.2064 & 0.491759 \\
Bilatfilt & 17.259 & 8.250404 & 16.254 & 0.498439 \\
Wiener & 19.28216 & 9.909887 & 14.2015 & 0.454266 \\
Bat algorithm (proposed) & 55.60916 & 45.72914 & 1.43606 & 0.9967 \\
Medfilt & 31.59428 & 21.71134 & 3.97919 & 0.941845 \\
Noise image & 14.7485 & 10.9870 & 30.8276 & 0.4501 \\
\hline
\end{tabular}




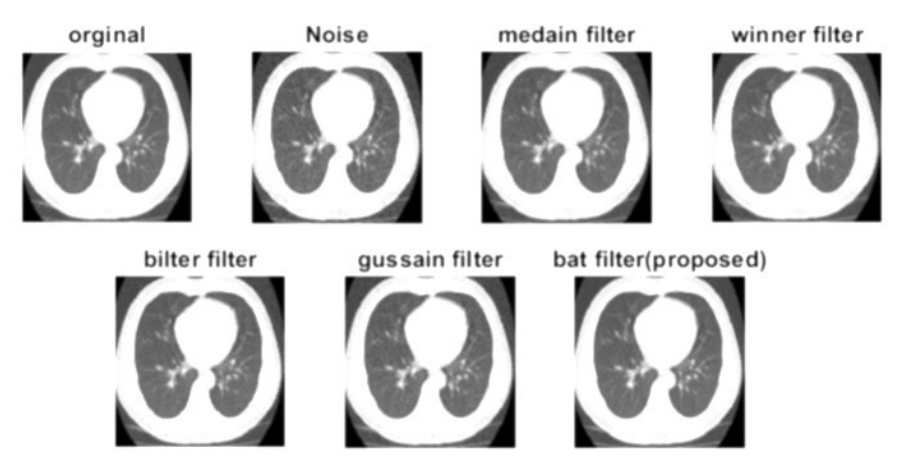

Figure 4: The CT medical image enhancement by proposed Bat algorithm and compare with other technique when adding poisson noise
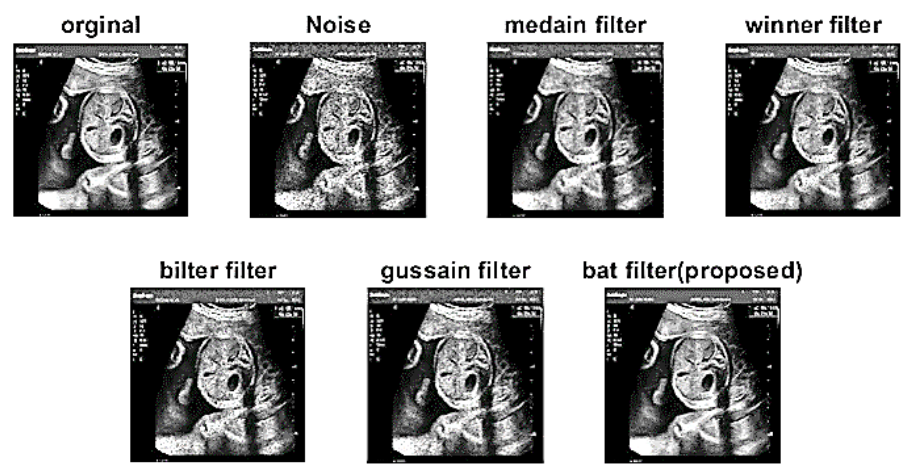

Figure 5: The ultrasound medical image enhancement by the proposed Bat algorithm and compare with other techniques when adding speckle noise

Table 3: The performance measures of the proposed method and compare with other techniques for speckle noise removal

\begin{tabular}{lllll}
\hline Filter & PSNR & SNR & MES & SSIM \\
\hline Gaussfilt & 26.52503 & 16.61562 & 11.2293 & 0.794528 \\
Bilatfilt & 25.02349 & 15.18409 & 12.447 & 0.797158 \\
Wiener & 26.78003 & 16.87542 & 10.2137 & 0.841703 \\
Bat algorithm (proposed) & 64.98037 & 54.96942 & 2.958578 & 0.996023 \\
Medfilt & 26.36113 & 16.29422 & 11.1503 & 0.79982 \\
Noise image & 16.7886 & 12.7212 & 25.2462 & 0.5521 \\
\hline
\end{tabular}

From Table II show the power of the proposed technique to de-noise the salt and pepper noise from a medical image when compared with other technique and the proposed method achieves 55.6 the value of PSNR but the second-best filter (median filter) achieves the 31.5 PSNR. From Table III show observe the power of the proposed technique to de-noise the speckle noise from a medical image when compared with other technique and the proposed method achieves 64.9 the value of PSNR but the second-best filter (Wiener filter) achieves the 26.7 PSNR. From Table IV show observe the power of the proposed technique to de-noise the Poisson noise from a medical image when compared with other technique and the proposed method achieves 63.6 the value of PSNR but the second-best filter (bilateral filter) achieves the 33.9 PSNR.

Table 4: The performance measures of the proposed method and compare with other techniques for speckle noise removal

\begin{tabular}{lllll}
\hline Filter & PSNR & SNR & MES & SSIM \\
\hline Gaussfilt & 26.52503 & 16.61562 & 11.2293 & 0.794528 \\
Bilatfilt & 25.02349 & 15.18409 & 12.447 & 0.797158 \\
Wiener & 26.78003 & 16.87542 & 10.2137 & 0.841703 \\
Bat algorithm (proposed) & 64.98037 & 54.96942 & 2.958578 & 0.996023 \\
Medfilt & 26.36113 & 16.29422 & 11.1503 & 0.79982 \\
Noise image & 16.7886 & 12.7212 & 25.2462 & 0.5521 \\
\hline
\end{tabular}


Table 5: The performance measures of the proposed method and compare with other techniques for Poisson noise removal

\begin{tabular}{lllll}
\hline Filter & PSNR & SNR & MES & SSIM \\
\hline Gaussfilt & 32.95095 & 23.09426 & 12.51218 & 0.913035 \\
Bilatfilt & 33.90705 & 24.07395 & 8.664130 & 0.941938 \\
Wiener & 33.27822 & 23.41648 & 11.02376 & 0.932075 \\
Bat algorithm (proposed) & 63.69802 & 53.86443 & 1.55461 & 0.999561 \\
Medfilt & 30.72685 & 20.80185 & 13.5447 & 0.90567 \\
Noise image & 19.7432 & 13.1234 & 23.2024 & 0.67642 \\
\hline
\end{tabular}

\section{Conclusions}

This work proposes the bat algorithm to de-noise the medical image with different types of noise. The proposed method, which achieves great results for enhancing the medical image. After that, a comparison is made between the method proposed with other enhancement techniques Gaussian filter, median filter, bilateral filter, and wiener filter. Most importantly, the proposed Bat optimization image de-noising achieves better RMSE and PSNR values than other techniques. The quality of images produced by using the proposed method on the corrupted images with Gaussian noise is compared concerning RMSE against the traditional enhancement technique. Based upon the experimental results, this method presents optimum values of RMSE for the images that are de-noised. Based upon the result, we observe the PSNR performance of the proposed method $(60.6,55.6,64.9,63.6)$ Gaussian noise, salt-and-pepper noise, speckle noise, and Poisson noise on order, but the median filter achieves PSNR (26.35, 31.59, 26.36, 30.72), but Gaussian filter achieves PSNR(24.27, 20.86, 26.52, 32.95), where the bilateral filter achieves PSNR $(23.74,17.25,25.02,33.90)$, where the Wiener filter achieves PSNR $(26.24,19.28,26.78,33.27)$ Gaussian noise, salt \& pepper noise, speckle noise On order.

Author contribution

All authors contributed equally to this work.

Funding

This research received no specific grant from any funding agency in the public, commercial, or not-for-profit sectors.

Data availability statement

The data that support the findings of this study are available on request from the corresponding author.

Conflicts of interest

The authors declare that there is no conflict of interest.

\section{Reference}

[1] Umbaugh, S. E. Digital Image Processing and Analysis Human and Computer Vision Applications with CVIPtools, second edition. 2021.

[2] H. S. Badi, S. Hussein, Hand posture and gesture recognition technology, Neural Comput. Appl., 25 (2014) 871-878. https://doi.org/10.1007/s00521-014-1574-4

[3] A. Mittal, H. Jindal, Novelty in Image Reconstruction using DWT and CLAHE, Int. j. image graph. Signal process., 5 (2017) 28-34 . https://doi.org/10.5815/ijigsp.2017.05.04

[4] F. Kallel, A. Ben Hamida, A New Adaptive Gamma Correction Based Algorithm Using DWT-SVD for Non-Contrast CT Image Enhancement, IEEE Trans. Nanobioscience, 16 (2017) 666-675. https://doi.org/10.1109/TNB.2017.2771350

[5] Z. Al-Ameen, Satellite image enhancement using an ameliorated balance contrast enhancement technique, Trait. du Signal, 37 (2020) 245-254, https://doi.org/10.18280/ts.370210

[6] B. K. Rajan, H. M. Harshan, G. Venugopal, Veterinary Image Enhancement using DWTDCT and Singular Value Decomposition, 2020 International Conference on Communication and Signal Processing (ICCSP), Chennai, India, 2020, 0920-0924. https://doi.org/10.1109/ICCSP48568.2020.9182414

[7] U. Acharya, S. K. undefined, Particle swarm optimized texture based histogram equalization (PSOTHE) for MRI brain image enhancement, Optik, 224(2020) 165760. https://doi.org/10.1016/j.ijleo.2020.165760

[8] Y. Chen, F. He, H. Li, D. Zhang, Y. Wu, A full migration BBO algorithm with enhanced population quality bounds for multimodal biomedical image registration, Appl. Soft Comput., 93 (2020) 106335. https://doi.org/10.1016/j.asoc.2020.106335

[9] P. Kandhway, A. K. Bhandari, A. Singh, A novel reformed histogram equalization based medical image contrast enhancement using krill herd optimization, Biomed. Signal Process. Control, 56 (2020), https://doi.org/10.1016/j.bspc.2019.101677 
[10] Dubey, H. et al. 2017. Fog Computing in Medical Internet-of-Things: Architecture, Implementation, and Applications, In: Khan, S., Zomaya, A., Abbas, A. (eds) Handbook of Large-Scale Distributed Computing in Smart Healthcare. Scalable Computing and Communications. Springer, pp. 281-321. Cham. https://doi.org/10.1007/978-3-319-58280-1_11

[11] A. M. Rahmani et al., Exploiting smart e-Health gateways at the edge of healthcare Internet-of-Things: A fog computing approach, Futur. Gener. Comput. Syst., 78 (2018) 641-658. https://doi.org/10.1016/j.future.2017.02.014

[12] L. T. Rasheed, A Comparative Study of Various Intelligent Controllers' Performance for Systems Based on Bat Optimization Algorithm, Eng. Technol. J., 38 (2020) 938-950. https://doi.org/10.30684/etj.v38i6A.622

[13] H. K. Chatterjee, R. Gupta, M. Mitra, A statistical approach for determination of time plane features from digitized ECG, Comput. Biol. Med., 41 (2011) 278-284. https://doi.org/10.1016/j.compbiomed.2011.03.003

[14] H. N. Abdullah , H. K. Abdul Jaleel, Deep CNN Based Skin Lesion Image Denoising and Segmentation using Active Contour Method, Eng. Technol. J., 37 (2019) 464-469. https://doi.org/10.30684/etj.37.11A.3

[15] P. Kora, S. R. Kalva, Improved Bat algorithm for the detection of myocardial infarction, Springer Plus, 4 (2015) 1-18. https://doi.org/10.1186/s40064-015-1379-7

[16] H. A. R. Akkar, S. A. Salman, Detection of Biomedical Images by Using Bio-inspired Artificial Intelligent, Eng. Technol. J., 38 (2020) 255-264. https://doi.org/10.30684/etj.v38i2A.319

[17] Yang, XS. 2010. A new metaheuristic Bat-inspired Algorithm, Studies in Computational Intelligence, vol. 284, pp. 65-74. Springer, Berlin, Heidelberg. https://doi.org/10.1007/978-3-642-12538-6_6

[18] A. Qayyum, S. M. Anwar, M. Awais, M. Majid, Medical image retrieval using deep convolutional neural network, Neurocomputing, 266 (2017) 8-20. https://doi.org/10.1016/j.neucom.2017.05.025

[19] X. S. Yang, A new metaheuristic Bat-inspired Algorithm, in Studies in Computational Intelligence, 284 (2010) 65-74. https://doi.org/10.1007/978-3-642-12538-6_6

[20] X.-S. Yang, S. Deb, Cuckoo Search via Lévy Flights, 2009 World Congress on Nature \& Biologically Inspired Computing (NaBIC), Coimbatore, India, 2009, 210-214. https://doi.org/10.1109/NABIC.2009.5393690

[21] X. X. Ma, J. S. Wang, Optimized Parameter Settings of Binary Bat Algorithm for Solving Function Optimization Problems, J. Electr. Comput. Eng., 2018 (2018) 9. https://doi.org/10.1155/2018/3847951

[22] Welcome to the Cancer Imaging Archive - The Cancer Imaging Archive (TCIA). https://www.cancerimagingarchive.net

[23] Brain Tumor Classification (MRI) | Kaggle. https://www.kaggle.com/sartajbhuvaji/brain-tumor-classification-mri

[24] CT Medical Images | Kaggle. https://www.kaggle.com/kmader/siim-medical-images 